\title{
Repercusiones del uso de Dispositivos de Avance Mandibular como Terapia para el Síndrome de Apnea Obstructiva del Sueño en el Complejo Temporomandibular. Revisión Narrativa
}

\author{
Repercussions of the use of Mandibular Advancement Devices as Therapy for Obstructive \\ Sleep Apnea Syndrome in the Temporomandibular Complex. Narrative Review
}

\author{
Valenzuela Chaigneau, Francisco; Field Seisdedos, Camila; \\ Ugarte Sánchez; Fernando \& Gracia Abuter, Benjamín
}

\begin{abstract}
VALENZUELA, C.,F.; FIELD, S. C.; UGARTE, S. F. \& GRACIA, A. B. Repercusiones del uso de dispositivos de avance mandibular como terapia para el síndrome de apnea obstructiva del sueño en el complejo temporomandibular. Revisión narrativa. Int. J. Odontostomat., 12(1):7-14, 2018.
\end{abstract}

RESUMEN: El objetivo de este trabajo fue escribir, según la literatura científica disponible más actual, los efectos que inducen el uso de dispositivos de avance mandibular, como terapia para el SAHOS, en el sistema temporomandibular de los pacientes. Se realizó una revisión de la literatura más actual (últimos 10 años; 2006-2016) a partir de la búsqueda electrónica en las bases de datos PubMed, TripData Base, Epistemonikos, The Cochrane Library y las revistas especializadas Journal of Clinical Sleep Medicine y SLEEP. Con el uso de las palabras clave: "Mandibular advancement device", "orthodonthic appliances", "sleep apnea syndroms", "sleep apnea obstructive", "Temporomandibular joints disorder", los operadores booleanos AND y OR. Se realizó un análisis crítico de la literatura evaluando nivel de evidencia, grado de recomendación y riesgo de sesgo de cada publicación. La búsqueda en las distintas bases de datos arrojó un total de 242 documentos, de los cuales 60 fueron seleccionados por título y abstract. Luego 8 estudios fueron descartados por estar repetidos. Se aplicaron los criterios de inclusión y exclusión quedando un total de 20 artículos; se eliminaron 8 por no responder a la pregunta de investigación y se añadió 1 título mediante la búsqueda manual. Finalmente, se analizaron 13 artículos; 2 revisiones sistemáticas, 2 ensayos clínicos aleatorizados y 6 series de casos. La mayoría de los documentos incluidos concuerda en que los efectos inducidos por los DAM, sobre el complejo temporomandibular son mínimos y reversibles, sin explicitar ningún diagnóstico de TTM en particular. Sin embargo, esta evidencia viene en su mayoría de estudios recomendables, pero no concluyentes. Se necesitan más y mejores estudios para realizar un análisis y abstraer conclusiones más certeras. Estos deben ser homogéneos a la hora de clasificar TTM y definir un protocolo óptimo de avance mandibular.

PALABRAS CLAVE: dispositivo de avance mandibular,aparatos ortodóncicos, síndrome de apnea durante el sueñom apnea del sueño obstructiva, trastornos de las articulaciones temporomandibulares.

\section{INTRODUCCIÓN}

El síndrome de apnea/hipoapnea obstructiva del sueño (SAHOS) es un desorden respiratorio del sueño caracterizado por la sucesión de múltiples eventos en dónde existe cese o disminución del flujo aéreo durante el sueño, con persistencia de esfuerzo ventilatorio (Quan \& Gillin, 1999). Se caracteriza por ronquidos a repetición, pausas respiratorias, y aumento del esfuerzo respiratorio, asociado a múltiples microdespertares en el transcurso del sueño.
Anatómicamente, se explica por un colapso de las vías aéreas superiores durante el sueño, que puede suceder distintos niveles, comprometiendo diferentes estructuras (Lee-Chiong, 2006).

Su prevalencia, en la población adulta, se distingue por sexo, afectando a un $2 \%$ de las mujeres y un $4 \%$ de los hombres adultos de mediana edad (Young et al., 1993). La incidencia de esta patología 
aumenta progresivamente con la edad, hasta llegar a un máximo alrededor de los 65 años, dónde se estabilizaría (Young et al., 2004).

Los factores de riesgo más relevantes para la aparición de SAHOS son la obesidad, el aumento de la circunferencia del cuello, el sexo masculino y algunas características cráneofaciales (macroglosia, micrognatia, la clase II esqueletal, la incompetencia labial, la respiración bucal, congestión nasal, vía aérea angosta) (Lee). Los hábitos patológicos como el consumo de cigarrillos y el abuso de alcohol, también se asocian a una mayor prevalencia de desórdenes respiratorios del sueño (Young et al., 2004).

El tratamiento gold standard para esta patología es el dispositivo de presión positiva continua de la vía aérea (CPAP) sin embargo, este genera severos problemas en cuanto a la adherencia al tratamiento por parte de los pacientes (Weaver et al., 2008; Spicuzza et al., 2015). Entre sus alternativas encontramos múltiples opciones; desde las más conservadoras como cambio de hábitos y estilo de vida (higiene del sueño, dieta, actividad física) hasta las alternativas quirúrgicas, las cuales pretenden ampliar la vía aérea. Las más utilizadas son, la uvulopalatofaringoplastía, amigdalectomía y la cirugía maxilofacial, la cual busca modificar la configuración anatómica de las vías aéreas superiores (Bloch, 2006; Panossian \& Avidan, 2009). Otra alternativa de tratamiento son los dispositivos intraorales, los cuales son poco invasivos, reversibles, sencillos de utilizar y con buenos resultados frente a SAHOS leve y moderada (Hoekema et al., 2004, 2008). Su diseño más común varía entre retenedores linguales, elevadores del velo y de avance mandibular (DAM) (Ahrens et al., 2011). Los DAM buscan ampliar la vía aérea localizando a la mandíbula en una posición más protruida de la fisiológica de reposo de los pacientes (Sandeep et al., 2011). Su fundamento biológico tiene origen en el músculo geniogloso de la lengua, ya que al desplazar su inserción en los procesos geni mandibulares, la lengua tendría más espacio para asentarse y su raíz se alejaría de la pared posterior de la faringe, aumentando su diámetro (Hans et al., 1997).

Generar una posición mandibular más protruida que la del paciente en reposo y tienen buena efectividad; sin embargo, existe controversia respecto a sus efectos secundarios, ya que hay estudios que han reportado casos de dolor, incomodidad y hasta disfunción articular y/o musculoesqueletal, y otros que afirman lo contrario.
El objetivo de este estudio es describir según la literatura científica disponible más actual, los efectos de los dispositivos de avance mandibular como terapia para el SAHOS en el sistema temporomandibular de los pacientes. De esta manera, analizar críticamente dicha información y poder dilucidar la persistente controversia entre el uso de estos aparatos y sus repercusiones en la articulación temporomandibular (ATM) y/o músculos masticatorios.

\section{MATERIAL Y MÉTODO}

Se realizó una búsqueda de la literatura disponible utilizando palabras claves: mandibular advancement device como término no MeSH y orthodontic appliances, sleep apnea syndroms, sleep apnea obstructive y temporomandibular joint disorders como términos $\mathrm{MeSH}$; junto con los operadores booleanos "AND y "OR".

Las bases de datos utilizadas fueron: PubMed, TripData Base, Epistemonikos y The Cochrane Library. A su vez, se realizó esta búsqueda en las revistas especializadas Journal of Clinical Sleep Medicine y SLEEP.

Los criterios de inclusión que los artículos debían cumplir para ser considerados como parte de este estudio fueron:

a. Estudios cuyos diseños sean ensayos clínicos, revisiones sistemáticas con o sin meta análisis y reportes de casos.

b. Estudios en humanos, independiente del sexo, etnia o edad de los pacientes.

c. Texto completo disponible.

d. Investigaciones disponibles en idioma inglés o español.

e. Estudios con diez años de antigüedad; publicados desde enero del 2006 hasta septiembre del 2016.

Los criterios de exclusión para los artículos fueron:

a. Estudios cuyos sujetos de investigación fueran animales.

b. Publicaciones diseñadas como reportes de como 
cartas al editor, opiniones de expertos, comentarios, o reportes de casos.

c. Investigaciones que utilicen otros aparatos intraorales para el uso del tratamiento de la apnea obstructiva del sueño; como retenedores linguales y elevadores del velo.

d. Estudios cuyos sujetos de investigación sean o incluyan pacientes con malformaciones esqueletales o patologías sindrómicas que puedan afectar la anatomía o función del sistema estomatognático.

El análisis crítico de la literatura fue realizado evaluando el nivel de evidencia, grado de recomendación y riesgo de sesgo de cada publicación incluida dentro de los resultados obtenidos. El nivel de evidencia y grados de recomendación fueron evaluados mediante las tablas propuestas por el Centre for Evidence-Based Medicine (Oxford Centre for Evidence-Based Medicine, 2018) de la Universidad de Oxford, expuestas en la Tabla I y la Tabla II, respectivamente. La Tabla III, expone el significado de cada grado de recomendación.

La evaluación de riesgo de sesgo en estudios clínicos, se realizó en base a la pauta proporcionada por "The Cochrane Collaboration", tomando en consideración los tipos de riesgos más frecuentes, excluyendo la última categoría "Otros sesgos". La evaluación fue clasificada en "Bajo riesgo", "Alto riesgo" o "Riesgo poco claro" de acuerdo al cumplimiento de las categorías, evaluadas con los siguientes símbolos y su significado ( $V$ : Adecuado; ?: Poco claro/ ineficiente; $¥$ : No se menciona) (Tabla IV).

Tabla I. Niveles de evidencia según CEBM.

\begin{tabular}{ll}
\hline $\begin{array}{l}\text { Nivel de } \\
\text { Evidencia }\end{array}$ & Tipo de estudio \\
\hline $1 a$ & Revisión sistemática den ensayos clínicos aleatorizados, con homogeneidad \\
$1 b$ & Ensayo clínico aleatorizado con intervalo de confianza estrecho \\
$1 c$ & Práctica clínica (todos o ninguno) \\
$2 a$ & Revisión sistemática de estudios de cohorte, con homogeneidad \\
$2 b$ & Estudio de cohortes o ensayo clínico aleatorizado de baja calidad \\
$2 c$ & "Outcomes" Research, estudios ecológicos \\
$3 a$ & Revisión sistemática de estudios de casos y controles, con homogeneidad \\
$3 b$ & Estudio de casos y controles \\
4 & Serie de casos o estudio de cohorte y de casos y controles de baja calidad \\
5 & Opinión de expertos sin valoración crítica, o basados en fisiología \\
\hline
\end{tabular}

Tabla II. Grados de recomendación según CEBM.

\begin{tabular}{cl}
\hline Grado de Recomendación & Nivel de evidencia \\
\hline$A$ & Estudios de nivel 1 \\
$B$ & Estudios de nivel 2-3, o extrapolación de estudios de nivel 1 \\
$C$ & Estudios de nivel 4, o extrapolación de estudios de nivel 2-3 \\
$D$ & Estudios de nivel 5, o estudios no concluyentes de cualquier nivel \\
\hline
\end{tabular}

*Extrapolaciones se refiere a cuando los datos se usan en una situación que tiene diferencias clínicas importantes que la original del estudio.

Tabla III. Significado de grados de recomendación según CEBM

\begin{tabular}{cl}
\hline Grado de Recomendación & Significado \\
\hline$A$ & Extremadamente recomendable \\
$B$ & Recomendación favorable \\
$C$ & Recomendación favorable, pero no concluyente \\
$D$ & Ni se recomienda ni se desaprueba \\
\hline
\end{tabular}


VALENZUELA, C., F.; FIELD, S. C.; UGARTE, S. F. \& GRACIA, A. B. Repercusiones del uso de dispositivos de avance mandibular como terapia para el síndrome de apnea obstructiva del sueño en el complejo temporomandibular. Revisión narrativa. Int. J. Odontostomat., 12(1):7-14, 2018.

Tabla IV. Riesgo de sesgo de los estudios.

\begin{tabular}{|c|c|c|c|c|c|c|c|c|}
\hline & & $\begin{array}{l}\text { Generación } \\
\text { de secuencia }\end{array}$ & $\begin{array}{l}\text { Oculta- } \\
\text { miento }\end{array}$ & $\begin{array}{c}\text { Cegamiento } \\
\text { paciente y } \\
\text { personal }\end{array}$ & $\begin{array}{l}\text { Cegamiento } \\
\text { evaluadores } \\
\text { de resultados }\end{array}$ & $\begin{array}{l}\text { Datos } \\
\text { resultados } \\
\text { completos }\end{array}$ & $\begin{array}{l}\text { Notifica- } \\
\text { ción no } \\
\text { selectiva }\end{array}$ & Total \\
\hline \multirow{10}{*}{ 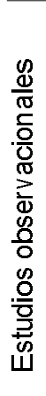 } & Autor, año & & & & & & & \\
\hline & Smith et al. (2014) & - & - & $\sqrt{ }$ & - & $\sqrt{ }$ & $\sqrt{ }$ & Moderado \\
\hline & Martınez-Gomis et al. (2010) & - & - & $\sqrt{ }$ & _- & $\sqrt{ }$ & $\sqrt{ }$ & Moderado \\
\hline & Giannasi et al. (2009) & - & _ & $\sqrt{ }$ & _ & - & $\sqrt{ }$ & Alto \\
\hline & Perez et al. (2012). & - & - & $\sqrt{ }$ & - & $\sqrt{ }$ & $\sqrt{ }$ & Moderado \\
\hline & Näpänkangas et al. (2012). & - & - & $\sqrt{ }$ & - & $\sqrt{ }$ & $\sqrt{ }$ & Moderado \\
\hline & Zhou et al. (2012). & _ & _ & $\sqrt{ }$ & _ & $\sqrt{ }$ & $\sqrt{ }$ & Moderado \\
\hline & Banhiran et al. (2014). & - & - & $\sqrt{ }$ & - & $\sqrt{ }$ & $\sqrt{ }$ & Moderado \\
\hline & Nakano et al. (2011) & - & - & $\sqrt{ }$ & - & $\sqrt{ }$ & $\sqrt{ }$ & Moderado \\
\hline & Aarab et al. (2009) & _ & _ & $\sqrt{ }$ & _ & $\sqrt{ }$ & $\sqrt{ }$ & Moderado \\
\hline$\varangle$ & Cunali et al. (2010) & $\overline{\sqrt{ }}$ & $\bar{?}$ & $\sqrt{ }$ & $\bar{v}$ & $\sqrt{ }$ & $\sqrt{ }$ & Bajo \\
\hline 㞻 & Doff et al. (2011). & $?$ & $?$ & $?$ & $?$ & $\sqrt{ }$ & $\sqrt{ }$ & Moderado \\
\hline
\end{tabular}

\section{RESULTADOS}

Luego de realizar el protocolo de búsqueda anteriormente señalado, se obtuvieron 242 documentos, los cuales se seleccionaron por título/abstract, y se eliminaron aquellos repetidos, y aquellos que no estuviesen publicados en español o inglés (por lo que estudios encontrados escritos en francés y chino fueron excluidos). Se aplicaron los criterios de inclusión/exclusión dejando un total de 12 artículos. Posteriormente, se agregó un título a la lista, proveniente de la búsqueda manual complementaria.

De entre los 13 artículos totales se distinguieron 2 revisiones sistemáticas, 2 ensayos clínicos aleatorizados y 9 series de casos. La selección de la búsqueda, está expresada en la Figura 1.

Con respecto al nivel de evidencia y grado de recomendación, se distinguieron 9 estudios con un nivel de evidencia 4 y uno con nivel $3 a$; lo que concuerda con un grado de recomendación $C$, información recomendable pero no concluyente. De los estudios restantes, 1 estudio tenía un nivel de evidencia 1a, y los otros dos niveles $1 \mathrm{~b}$; estos tres representan un grado de recomendación $\mathrm{A}$, por lo que es información extremadamente recomendable.
Para el análisis del riesgo de sesgo se incluyeron los estudios cuyos diseños fueran ECA u observacionales, excluyendo así las revisiones sistemáticas. Como resultado se obtuvieron: 1 estudio con alto riesgo, 9 con moderado riesgo de sesgo y 1 con bajo riesgo de sesgo. Estos resultados están expuestos en las Tablas $\mathrm{V}$ y VI.

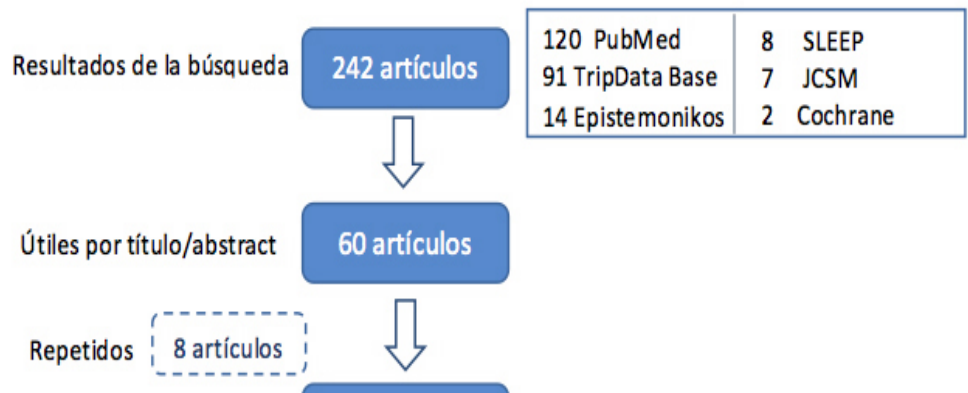

52 artículos

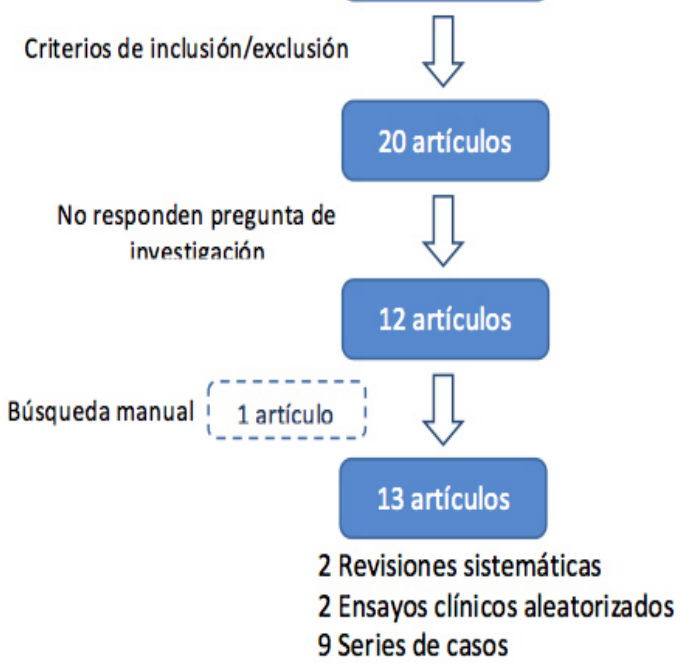

Fig. 1. La selección de la búsqueda bibliográfica. 


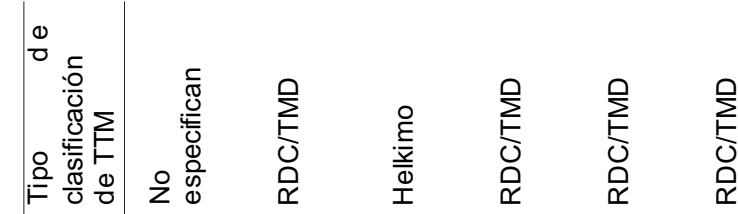

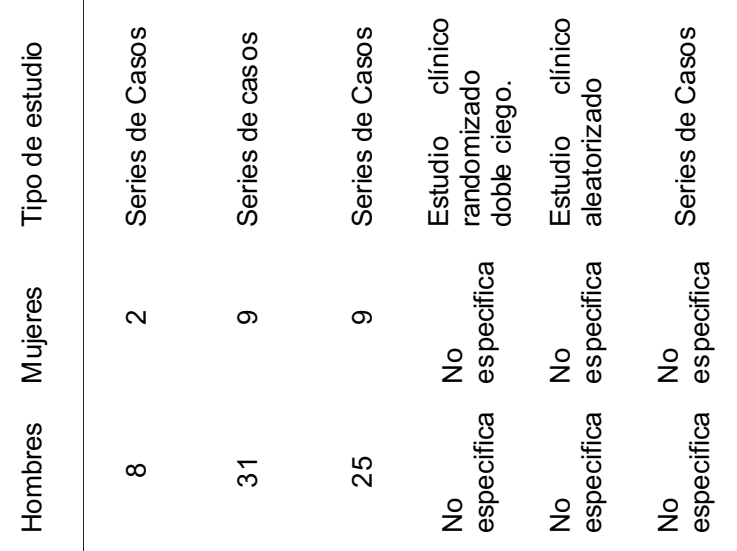

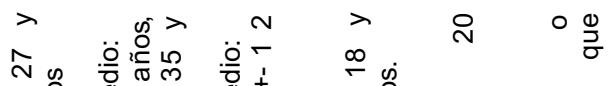

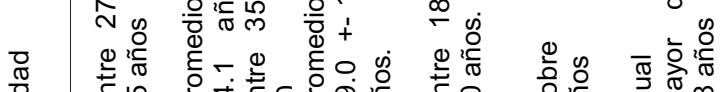

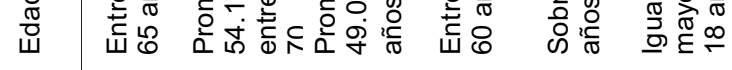

\begin{tabular}{|c|c|c|c|}
\hline 으 & 우 & 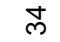 & $\sigma$ \\
\hline
\end{tabular}

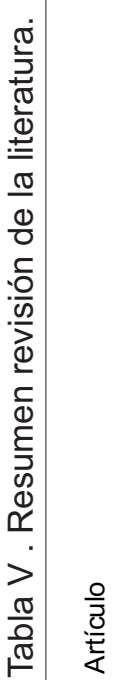

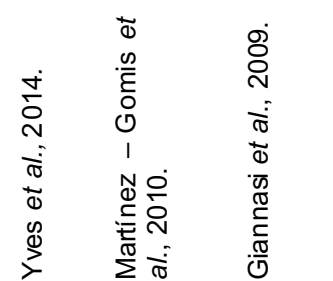

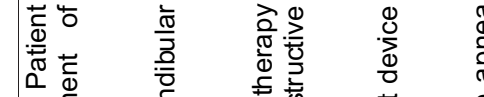

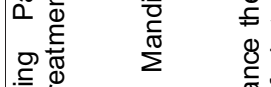

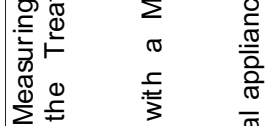

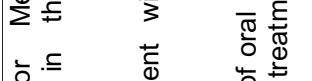

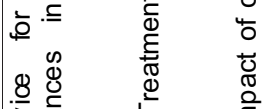

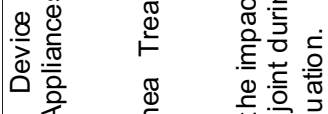

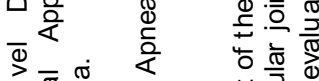$$
\text { 인 }
$$

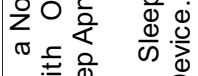$$
4 \frac{\pi}{0} \text { ठ }
$$

능

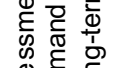

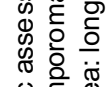

$$
\begin{aligned}
& \text { 을 } \\
& \text { 迥 }
\end{aligned}
$$

萡

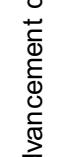

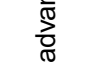

元 离

बํ.

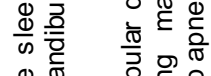

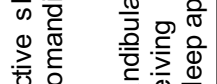

등 $\frac{\omega}{0}$ त

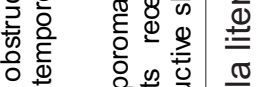

읗 .

듈

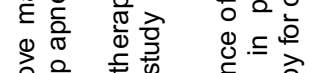

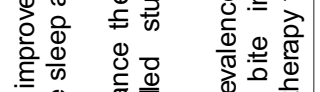

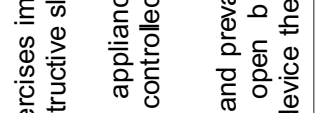

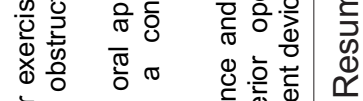

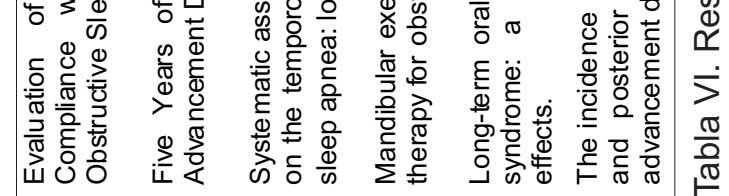

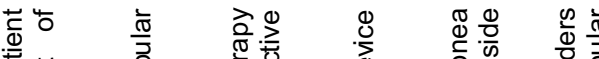

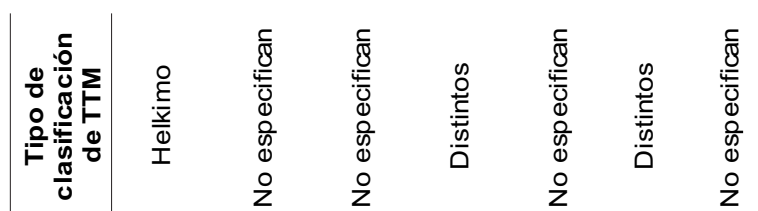

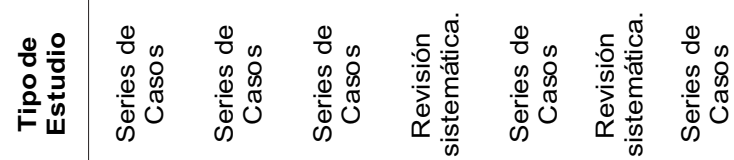

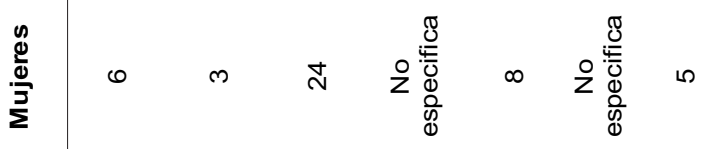

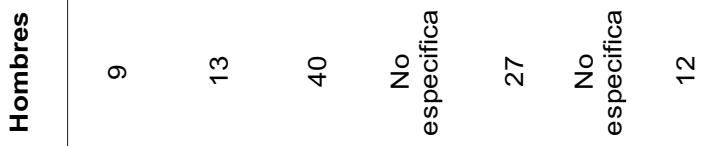

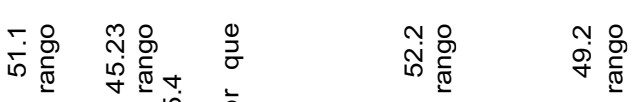

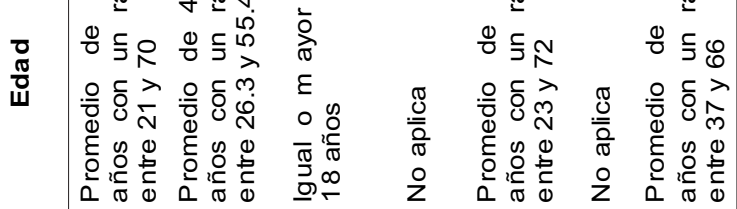

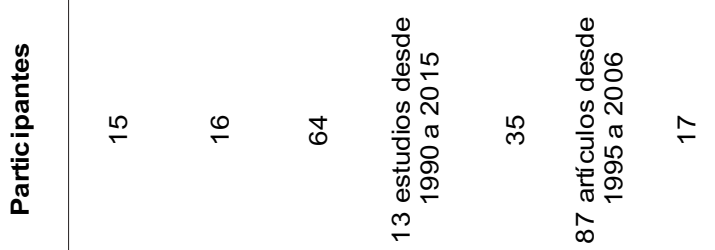

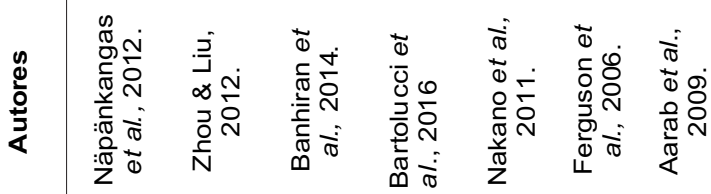

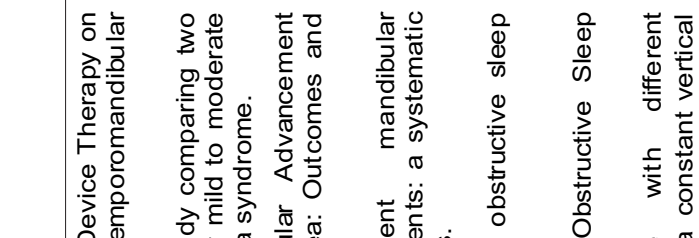

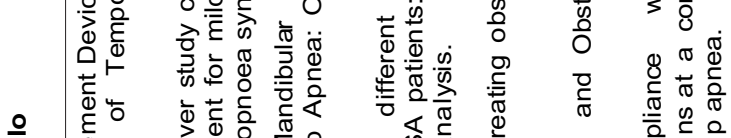

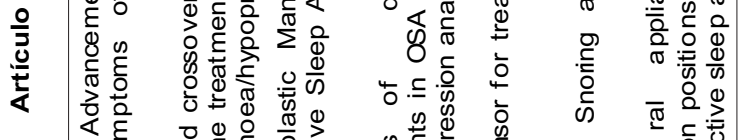

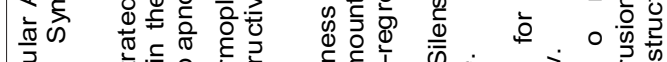

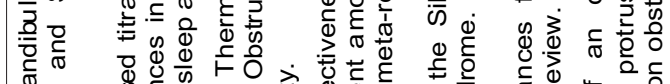

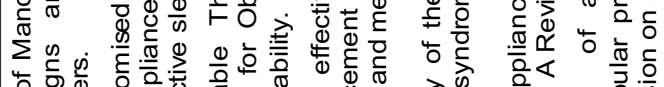

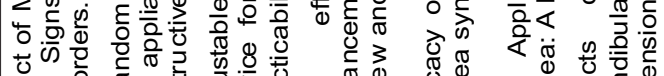

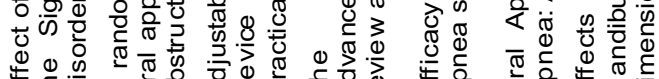

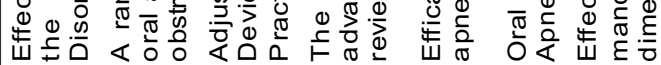




\section{DISCUSIÓN}

En esta revisión se seleccionaron 13 artículos, 9 Series de Casos, 2 estudios clínicos randomizados y 2 revisiones sistemáticas. Por otra parte, cabe mencionar la limitación de idiomas de las publicaciones, ya que bajo este criterio sólo fueron incluidos aquellos estudios que estuviesen publicados en español o inglés; por lo que estudios escritos en otros idiomas (francés, chino) fueron excluidos.

De estos se identificó en su mayoría una recomendación favorable pero no concluyente; es decir, son publicaciones con un moderado grado de recomendación y bajo nivel de evidencia correspondientes a los estudios observacionales (Series de Casos). En estos se tomaron grupos de pacientes con un promedio total de 44 participantes, siendo el estudio de Perez et al. (2013), en el que tuvo una mayor muestra (167 individuos).

Estos estudios, en general, analizaron pacientes adultos (mayor de 18 años) que padecían SAHOS y su tratamiento fue realizado con dispositivos de avance mandibular, para determinar los efectos positivos y negativos en el tiempo. En la mayoría de ellos no se indicó el método diagnóstico para los TTMs y los síntomas se describen como molestias o sensaciones. De los que definieron sus métodos diagnósticos, lo hicieron en base al método de Helkimo y RCD/TMD.

En cuanto al desplazamiento mandibular, no se observó un consenso en el protocolo de protrusión mandibular óptima entre los estudios, que por lo general iba desde el $25,50,75 \%$ y protrusión máxima. En un estudio que evaluó distintos porcentajes de protrusión $(25,50,75 \%)$, el número de efectos adversos fue mayor después del $50 \%$ de protrusión, y los AHI (Índice de Apneas/Hipoapneas) a ese porcentaje fueron los menores; por lo que se recomendaría realizar los tratamientos no sobrepasando el $50 \%$ de avance mandibular. Con respecto a efectos adversos asociados a TTM, estos fueron transitorios y mínimos, específicamente representados por la sensibilidad en la región del músculo masetero por las mañanas (Aarab et al., 2010).

Estos datos se relacionan con lo presentado por Martínez-Gomis et al., donde 41 pacientes con SAHOS fueron tratados con DAM, usado todas las noches durante 5 años. Antes del tratamiento todos los pacientes fueron sometidos a un examen dental aplicando el RCD/ TMD, y se catalogaron en un grupo sin TTMs y otros 3 grupos con TTM (I: dolor miofascial, II: desplazamiento discal y artralgia, III: artritis yosteoartrosis). Quince pacientes completaron el estudio de 5 años y de ellos, 10 que en un principio fueron diagnosticados con TTM a través del RCD/TMD ( 3 con dolor miofascial, 7 con desplazamiento discal y 2 con artralgia), solo 1 permaneció con DDCR. Por lo tanto, este estudio concluyó que el uso de DAM no afecta significativamente la prevalencia de los TTM (Martínez-Gomis et al., 2010).

En otra publicación de series de casos de Giannasi et al. (2009), se usó el método diagnóstico de Helkimo (Anamnestic Dysfunction Index) y se concluyó que el uso a largo plazo de DAM para pacientes con bruxismo y SHAOS no agravan los síntomas de TTMS, por el contrario, los alivió en los casos que los pacientes presentaban TTMs moderados.

En Kentucky (EEUU), Pérez y colaboradores realizaron un estudio retrospectivo en donde se analizaron antecedentes de 167 pacientes que padecían SAHOS, y se concluyó que el uso de los dispositivos de avance mandibular podría desarrollar TTMs en un número menor de pacientes, sin embargo, estos signos son transitorios (Pérez et al., 2013).

Otro estudio, realizado por Banhiran et al. (2014), fueron seleccionados 64 pacientes, su objetivo fue analizar los resultados (eficacia, efectos adversos y calidad de vida) del tratamiento con DAM. No se menciona la clasificación diagnóstica usada para los TTMs antes del estudio, por lo que no se sabe que diagnóstico previo tenían estos pacientes. Con respecto a la sintomatología, cuatro no siguieron el estudio por molestias en la ATM, siendo que un criterio de exclusión al seleccionar los pacientes era que no tuvieran TTMs y tres pacientes tuvieron un malestar severo por el que no pudieron seguir el tratamiento (Banhiran et al.).

De acuerdo a los estudios clínicos randomizados, se observó un grado de recomendación A (extremadamente recomendables). El promedio de pacientes que participaron en estos estudios fue de 40 , divididos en grupos placebo y otros con tratamiento.

Cunali et al. (2010) realizaron un estudio clínico randomizado donde pacientes adultos con SAHOS moderado y presencia de TTMs según el RDC/TMD fueron sometidos a tratamiento con dispositivos de avance mandibular. Los pacientes se dividieron en un grupo placebo (PT) y otro con terapia de ejercicios (ST) para TTMs. En este último se observó que los ejercicios mandibulares permiten a los pacientes con TTMs usar 
dispositivos de avance mandibular demostrando ser efectivos en la reducción del dolor y aumento en la adherencia al tratamiento produciendo una mejora en calidad de sueño y vida (Cunali et al.).

En otro estudio clínico, con el mismo nivel de evidencia, publicado por Doff et al. (2013), se seleccionaron 2 grupos, uno de 51 pacientes con aparatos intraorales (avance mandibular) y otro de 52 pacientes con CPAP. Sus resultados mostraron que la ocurrencia de los TTMs se incrementó durante el periodo inicial en el grupo con dispositivo de avance mandibular, pero después de 2 años tendió a volver a los mismos valores que al inicio del estudio. En los pacientes con SAHOS la terapia con DAM resulta en un aumento de dolor en el complejo temporomandibular en comparación con la terapia del CPAP, pero no causa limitaciones funcionales. Estos resultados sugieren que un posible desarrollo de TTMs o dolor temporal en el complejo temporomandibular no son una contraindicación para el uso de dispositivos de avance mandibular en pacientes con SAHOS (Doff et al.).

Finalmente, una de las revisiones sistemáticas evaluadas demostró ser extremadamente recomendable. En ella se sugiere que los DAM, al forzar la mandíbula a una posición anterior, elongan las fibras de los músculos elevadores y el ligamento temporomandibular causando tensión en ellos y en los tejidos retrodiscales, produciendo dolor en las estructuras temporo-mandibulares (Bartolucci et al., 2016). Otra revisión clasificó las complicaciones en dos grupos, uno donde eran menores, transitorias y otro, donde eran más severas y permanentes. Los efectos adversos menores y transitorios incluyeron excesiva salivación, malestar muscular y articular. Pero las complicaciones más severas y permanentes de TTMs raramente ocurrían (Ferguson et al., 2006).

\section{CONCLUSIÓN}

Conocer las repercusiones potencialmente perjudiciales de los DAM sobre el complejo temporomandibular es de gran interés científico, ya que esta es una alternativa real, eficiente y con gran aceptabilidad entre los pacientes que padecen SAHOS; y es necesario comprobar su seguridad y controlar sus efectos adversos.

A partir de lo investigado, podemos concluir que la mayoría de los estudios incluidos concuerda en que los efectos inducidos por los DAM sobre el complejo temporomandibular son mínimos, reversibles y se dan principalmente en un periodo inicial de adaptación. Sin embargo, estos son mitigables gracias a algunos ejercicios mandibulares y control periódico con el dentista, e impiden la aparición de efectos adversos más severos y permanentes.

A su vez, debemos tener en cuenta que un gran porcentaje de los estudios son de baja calidad, con evidencia recomendable pero no concluyente y se deben tomar sus conclusiones con precaución. Por lo tanto, para abordar de una manera óptima el tema, se necesitan más estudios, de mejor calidad, con mejor diseño y con mayor potencia estadística y nivel de evidencia, los cuales deben enfocarse más en la relación entre el mecanismo de acción de los DAMs y sus repercusiones en el complejo temporomandibular. Esto permitiría establecer un protocolo de acción óptimo (grado de protrusión, frecuencia y duración), independiente del diseño del aparato.

Además, los estudios deberían siempre apuntar a diagnosticar los TTM con criterios claros y reproducibles, y en conjunto con exámenes complementarios cuando el caso lo requiera. Con esto, se podría realizar un diagnóstico certero de TTM previo al inicio de la terapia con DAM, y así determinar la condición basal de los pacientes y luego observar su evolución. De este modo, se podrían realizar asociaciones reales, con el peso suficiente como para orientar al profesional en sus decisiones clínicas y mejorar la predicción de éxito según las características de cada paciente.

VALENZUELA, C. F.; FIELD, S. C.; UGARTE, S. F. \& GRACIA, A. B. Repercussions of the use of mandibular advancement devices as therapy for obstructive sleep apnea syndrome in the temporomandibular complex. Narrative review. Int. J. Odontostomat., 12(1):7-14, 2018.

ABSTRACT: The aim of this study was to describe, based on the most recent scientific literature available, the effects produced by the mandibular advance appliances (MAA) as a therapy for obstructive sleep apnea-hypopnea syndrome (OSAHS) in the temporomandibular system. We carried out a review of the most current literature published in the last 10 years, based on an electronic search in PubMed, TripData Base, Epistemonikos, The Cochrane Library and the specialized magazines Journal of Clinical Sleep Medicine and SLEEP. The key words used for each search were "MANDIBULARADVANCEMENT DEVICE", "ORTHODONTIC APPLIANCES", "SLEEP APNEA SYNDROMS", "SLEEP APNEA, OBSTRUCTIVE", and "TEMPOROMANDIBULAR JOINT DISORDERS" combined with boolean operators AND 
and OR. A critical analysis of the literature was evaluated based on the level of evidence, degree of recommendation and risk of bias of each publication. We obtained 242 articles and 60 of these were selected by title and abstract. Inclusion and exclusion criteria were applied, obtaining 20 articles of which 8 were excluded because they did not answer the investigation question. One article was obtained by manual search. Of this number, 13 articles, 2 systematic reviews, 2 randomized clinical trial and 6 cases series were analyzed. Most of the articles analyzed agreed that the effects produced by the MAA in the temporomandibular complex are minimal and reversible, and they did not specify any TMD diagnosis in particular. However, this evidence comes mostly from recommended but inconclusive studies. More and better designed studies are needed, with homogeneous classification of TMD diagnostic criteria that allows to define an optimal protocol for mandibular advancement as a therapy.

KEY WORDS: Mandibular advancement device, Orthodonthic appliances, Sleep apnea syndroms, Sleep apnea obstructive, Temporomandibular joints disorder.

\section{REFERENCIAS BIBLIOGRÁFICAS}

Aarab, G.; Lobbezoo, F.; Hamburger, H. L. \& Naeije, M. Effects of an oral appliance with different mandibular protrusion positions at a constant vertical dimension on obstructive sleep apnea. Clin. Oral Investig., 14(3):339-45, 2010.

Ahrens, A.; McGrath, C. \& Hägg, U. A systematic review of the efficacy of oral appliance design in the management of obstructive sleep apnoea. Eur. J. Orthod., 33(3):318-24, 2011.

Banhiran, W.; Kittiphumwong, P.; Assanasen, P.; Chongkolwatana, C. \& Metheetrairut, C. Adjustable thermoplastic mandibular advancement device for obstructive sleep apnea: outcomes and practicability. Laryngoscope, 124(10):2427-32, 2014.

Bartolucci, M. L.; Bortolotti, F.; Raffaelli, E.; D'Antò, V.; Michelotti, A. \& Alessandri Bonetti, G. The effectiveness of different mandibular advancement amounts in OSA patients: a systematic review and meta-regression analysis. Sleep Breath, 20(3):911-9, 2016.

Bloch, K. E. Alternatives to CPAP in the treatment of the obstructive sleep apnea syndrome. Swiss Med. Wkly., 136(17-18):261-7, 2006.

Cunali, P. A.; Almeida, F. R.; Santos, C. D.; Valdrichi, N. Y.; Nascimento, L. S.; Dal-Fabbro, C.; Tufik, S. \& Bittencourt, L. R. Mandibular exercises improve mandibular advancement device therapy for obstructive sleep apnea. Sleep Breath, 15(4):717-27, 2010.

Doff, M. H.; Finnema, K. J.; Hoekema, A.; Wijkstra, P. J.; de Bont, L. G. \& Stegenga, B. Long-term oral appliance therapy in obstructive sleep apnea syndrome: a controlled study on dental side effects. Clin. Oral Investig., 17(2):475-82, 2013.

Ferguson, K. A.; Cartwright, R.; Rogers, R. \& Schmidt-Nowara, W. Oral appliances for snoring and obstructive sleep apnea: a review. Sleep, 29(2):244-62, 2006.

Giannasi, L. C.; Almeida, F. R.; Magini, M.; Costa, M. S.; de Oliveira, C. S.; de Oliveira, J. C.; Kalil Bussadori, S. \& de Oliveira, L. V. Systematic assessment of the impact of oral appliance therapy on the temporomandibular joint during treatment of obstructive sleep apnea: long-term evaluation. Sleep Breath, 13(4):375-81, 2009.

Hans, M. G.; Nelson, S.; Luks, V. G.; Lorkovich, P. \& Baek, S. J. Comparison of two dental devices for treatment of obstructive sleep apnea syndrome (OSAS). Am. J. Orthod. Dentofacial Orthop.,
111(5):562-70, 1997

Hoekema, A.; Stegenga, B. \& De Bont, L. G. Efficacy and co-morbidity of oral appliances in the treatment of obstructive sleep apneahypopnea: a systematic review. Crit. Rev. Oral Biol. Med., 15(3):137-55, 2004.

Hoekema, A.; Stegenga, B.; Wijkstra, P. J.; van der Hoeven, J. H.; Meinesz, A. F. \& de Bont, L. G. Obstructive sleep apnea therapy. J. Dent. Res., 87(9):882-7, 2008.

Lee-Chiong, T. L. Sleep. A Comprehensive Handbook. Hoboken, WileyLiss, 2006.

Martínez-Gomis, J.; Willaert, E.; Nogues, L.; Pascual, M.; Somoza, M. \& Monasterio, C. Five years of sleep apnea treatment with a mandibular advancement device. Side effects and technical complications. Angle Orthod., 80(1):30-6, 2010.

Oxford Centre for Evidence-Based Medicine (CEBM). Oxford Centre for Evidence-based Medicine - Levels of Evidence (March 2009). CEBM, 2018. Disponible en: https://www.cebm.net/2009/06/oxfordcentre-evidence-based-medicine-levels-evidence-march-2009/

Panossian, L. A. \& Avidan, A. Y. Review of sleep disorders. Med. Clin. North Am., 93(2):407-25, 2009.

Perez, C. V.; de Leeuw, R.; Okeson, J. P.; Carlson, C. R.; Li, H. F.; Bush, H. M. \& Falace, D. A. The incidence and prevalence of temporomandibular disorders and posterior open bite in patients receiving mandibular advancement device therapy for obstructive sleep apnea. Sleep Breath, 17(1):323-32, 2013.

Quan, S. F. \& Gillin, J. C. New definitions of sleep disordered breathing-Not yet a mandate for change in clinical practice. Sleep, 22(5):662, 1999.

Sandeep, C.; Gopinadh, A.; Babu, M. S. \& Ravuri, K. Revival of the eclipsed: The 5th dimension of a prosthodontist. Int. J. Dent. Clin., 3(1):71-4, 2011.

Spicuzza, L.; Caruso, D. \& Di Maria, G. Obstructive sleep apnoea syndrome and its management. Ther. Adv. Chronic Dis., 6(5):27385, 2015.

Weaver, T. E. \& Grunstein, R. R. Adherence to continuous positive airway pressure therapy: the challenge to effective treatment. Proc. Am. Thorac. Soc., 5(2):173-8, 2008.

Young, T.; Palta, M.; Dempsey, J.; Skatrud, J.; Weber, S. \& Badr, S. The occurrence of sleep-disordered breathing among middle-aged adults. N. Engl. J. Med., 328(17):1230-5, 1993.

Young, T.; Skatrud, J. \& Peppard, P. E. Risk factors for obstructive sleep apnea in adults. JAMA, 291(16):2013-6, 2004.

Zhou, J. \& Liu, Y. H. A randomised titrated crossover study comparing two oral appliances in the treatment for mild to moderate obstructive sleep apnoea/hypopnoea syndrome. J. Oral Rehabil., 39(12):91422, 2012.

Dirección para correspondencia:

Francisco Valenzuela Chaigneau

Clínica San Damián, Estoril 50

Oficina 614

Las Condes

Santiago

CHILE

Email: drfvalenzuelach@gmail.com

Recibido : 10-07-2017

Aceptado: 05-09-2017 